\title{
Genetic bases of tomato marker-assisted selection in Belarus
}

\author{
Olga G. Babak, Natalia A. Nekrashevich, Konstantsia K. Yatsevich, Sergey V. Malyshev \\ and Alexander V. Kilchevsky*
}

\begin{abstract}
To develop tomato hybrids and varieties with a high fruit quality, we gradually solved the following tasks: development of DNAmarking methods for long shelf life genes; the genes modifying the biosynthesis of carotenoids and their composition; testing of the elaborated methods on the developed breeding material; the selection of samples with different allelic composition of fruit quality genes; the development of $F_{1}$ hybrids using the method of successive crosses and their study; the selection of tomato forms by DNA-typing methods with target genes in $\mathrm{F}_{2}$ populations to develop valuable breeding samples; the study of carotenoids' accumulation peculiarities and their inheritance.

We used DNA-identification methods for fruit quality genes: $n o r$, rin, $n o r^{A}$ (long shelf life), $B, o g^{c}, h p 2^{d g}, g f-3$ (carotenoid content). The tomato hybrids, combining two pigment content genes and one long shelf life gene and the model forms with different

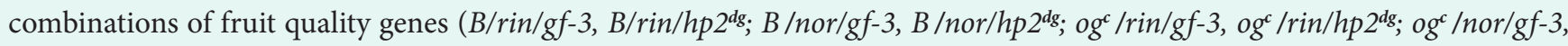
$o g^{c} /$ nor $\left./ h 2^{d g}\right)$ in a homozygous state were developed.

Use of the developed accessions with carotenoid content genes $\left(\operatorname{og} \mathrm{g} / h p 2^{d g}, B / h p 2^{d g}\right)$ as maternal forms and the accessions with complex fruit quality genes $\left(o g^{c} / h p 2^{d g} / n o r, o g^{c} / h p 2^{d g} /\right.$ rin, $B / h p 2^{d g} /$ nor, B/hp $2^{d g} /$ rin, og $/ g f-3 /$ nor, og $/ g f-3 /$ rin, B/gf-3/rin, B/gf-3/ nor) as paternal forms for hybridization contributes to high accumulation of carotenoids and a lond period of fruit storability.
\end{abstract}

Keywords: tomato, DNA-marker, fruit quality breeding

Laboratory of Ecological Genetics and Biotechnology, the Institute of Genetics and Cytology, NAS of Belarus, Minsk, Belarus

${ }^{*}$ Corresponding author: A. V. Kilchevsky E-mail: kilchev@presidium.bas-net.by

DOI: 10.2478/ebtj-2018-0017

(C) 2018 Authors. This work was licensed under the Creative Commons AttributionNonCommercial-NoDerivs 3.0 License.

\section{Introduction}

Carotenoids constitute a basis for biologically active substances of tomato, which along with low caloric content ensure the tomato usage as a product for functional nutrition that aids in the prevention of cancer, reduces the risk of cardiovascular diseases, atherosclerosis, increased immunity, the synthesis of visual pigments, and the activation of metabolic processes in a person due to their use. In this regard, the quality of the fruit (the content of biologically active substances and technological properties) is an important direction in tomato breeding.

In classical breeding, the forms with a varied fruit coloration are used in the development of varieties and hybrids with the accumulation of various carotenoids. However, the phenotypic assessment of the breeding material by the fruit color is insufficient in the selection of targeted forms (forms of interest) since it is impossible to differentiate a number of accumulated carotenoids with different biological values. This makes it possible to apply DNA-typing methods for the breeding material. The use of marker-assisted selection is very efficacious in both the initial lines' selection for hybridization and the subsequent analysis of the hybrid material and varieties. MarkerAssisted Selection (MAS) methods in tomato are widely used to develop cultivars with high biochemical composition, particularly carotenoids.

Along with biochemical characteristics of fruits, technological ones are important allowing to preserve high quality of products for a long term period by increasing the ripening period. 
Observed at the phenotypic level, interspecific and intraspecific diversity by coloration characters is associated with the polymorphism of structural genes controlling the synthesis and sequential development of organic molecules into terminal pigment compounds, as well as regulatory genes determining the expression level of certain gene groups. We should note that the regulation of carotenoid accumulation is very complicated and not fully understood. At present, it is the subject matter of many scientific centers. In the world breeding, a number of genes that control the content of fruit carotenoids were identified in tomato. More than 100 tomato genes associated with the maturation and accumulation of carotenoids were described. Most of these genes can be found in the databases of tomato genetic sequences. Our article (1) provides an overview of different gene groups that determine the fruit color.

The genes that control the ripening of tomato fruits (ripening inhibitor, nonripening, alcobaca) are most widely used in breeding. These genes are of considerable value in the practical breeding of tomato for storability since they have a sufficiently pronounced phenotypic effect in the heterozygote that allows to significantly improve commercial and technological qualities of tomato fruits without substantially impairing the biochemical composition. Regulatory genes used in breeding for the increased carotenoid content include mutations of lightdependent transcription factors, as well as mutations involved in the synthesis of carotenoids and chlorophyll degradation. A combination of these genes with a number of structural ones (tangerine, Beta, old-gold, etc.) determining the biosynthesis of carotenoids allows to develop the forms with high taste and technological qualities.

The aim of our research was to elaborate methods for molecular marking of alleles that affect the maturation and accumulation of carotenoids on the basis of database sequences and the identified polymorphism for studying of carotenoids' accumulation and their inheritance peculiarities to develop varieties and hybrids with high biochemical and technological qualities.

\section{Materials and Methods}

Depending on the stage of work, we used the following material for our research: the sample collections that belong to the Belarusian selection, C.M. Rick Tomato Genetics Resource Center (California); the hybrids of $\mathrm{F}_{1}$ forms selected in $\mathrm{F}_{2-3}$ populations that combine two pigment content genes and one long shelf life gene, (B/rin/gf, B/rin/hp; B /nor/gf, B/nor/hp; $\circ g^{c} / \mathrm{rin} / g f, \mathrm{og}^{c} / \mathrm{rin} / \mathrm{hp} ; \mathrm{og}^{c} / \mathrm{nor} / g f, \mathrm{og}^{c} /$ nor $/ \mathrm{hp}$ ) in a homozygous state.

Marker development. We have designed co-dominant Sequence Characterized Amplified Region (SCAR) and derived Cleaved Amplified Polymorfic Sequences (dCAPS) markers on the basis of GeneBank sequence and our own sequence data to detect gene quality polymorphism in the tomato collection.

Sequencing analysis. For sequencing, the amplification fragments were separated in $1.0 \%$ agarose gel and then excised and purified using the Agarose Gel Extraction Kit (Jena Bioscience) following the manufacturer's procedure. Sequencing reactions were performed using a set of Big Dye Terminator v 3.1 Cycle Sequencing Kit (Applied Biosystems). Sequencing reaction products were purified using columns Centri-Sep $^{\text {Tw }}$ (Applied Biosystems), dried under vacuum, dissolved in $20 \mu \mathrm{l}$ of formamide, denatured by heating at $95^{\circ} \mathrm{C}$ for 2 minutes and then performed capillary electrophoresis using a DNA sequencer -ABI PRISM ${ }^{\mathrm{TN}} 3500$ Genetic Analyzer (Applied Biosystems). Computer processing of the data obtained from sequencing was performed using the Sequencing Analysis Software v 5.2 (Applied Biosystems).

DNA-typing. DNA was extracted using the Genomic DNA Purification Kit (Thermo Scientific) of different tomato varieties seedlings as per manufacturer's instructions.

PCR was performed in $15 \mu \mathrm{l}$ : $50 \mathrm{ng}$ genomic DNA as a template, $0.2 \mathrm{mM}$ of each deoxynucleotide, $0.25 \mu \mathrm{M}$ of each primer, $7.5 \mu \mathrm{l} 2 \mathrm{x}$ Replication-Cycle Reaction (RCR) bufer, 0.4U Tornado Polymerase (Primetech, Belarus). The PCR parameters were: initial denaturation at $95^{\circ} \mathrm{C}$ for $15 \mathrm{~min}$. followed by 35 cycles of denaturation at $99^{\circ} \mathrm{C}$ for $1 \mathrm{~s}$, annealing at $47-55^{\circ} \mathrm{C}$ for $30 \mathrm{~s}$ and extension at $72^{\circ} \mathrm{C}$ for $30 \mathrm{~s}$. A final extension was at $72^{\circ} \mathrm{C}$ for $2 \mathrm{~min}$. The PCR reaction was performed in the Thermocycler Biometra T Professional Basic (Germany).

The PCR products for markers $h p-2^{d g}$, nor ${ }^{A}$, rin were separated by electrophoresis in a $1.5 \%$ agarose gel in the presence of etidium bromide and documented using BioRad GelDoc 2000 system (USA). Amplified fragment sizes were determined by using $100 \mathrm{bp}$ Plus DNA ladder (Thermo Scientific) as a molecular weight marker.

The PCR products for markers $B, o g^{c}, g f-3$, nor, were separated by electrophoresis in a polyacrylamide gel or on an automatic sequencer - Applied Biosystems Genetic Analyzer 3500. The sizes of the amplified fragments were determined using the S450 size standard.

Biochemical analysis. Tomato plants were grown in glazed greenhouses. The pigment composition analysis of tomato fruits was carried out by spectrophotometry and HPLC techniques in the Laboratory and Analytical Center for assessing the quality of new varieties and hybrids of vegetable crops during storage and processing at the All Russian Research Institute of Vegetable Breeding and Seed Production (http://www. vniissok.ru/stati/ispytatelnyy-centr-vniissok). The content of the following pigments was evaluated: lycopene, $\beta$-carotene, lutein, neurosporin, $\zeta$-каротин,chlorophyll.

\section{Results}

To identify the polymorphism of structural carotenoid biosynthesis genes, we developed markers to the tangerine $(t)$ allele of the carotenoid isomerase gene (CRTISO) and Beta carotene $(B)$ and old-gold crimson $\left(\mathrm{og}^{c}\right)$ alleles of Chloroplastspecific lycopene-b-cyclase (CYC-B).

The tangerine $(t)$ mutation in the gene encoding carotenoid isomerase (CRTISO) violates the stage of prolycopene (tetra-cislycopene) transformation into lycopene (trans-forms) and leads 


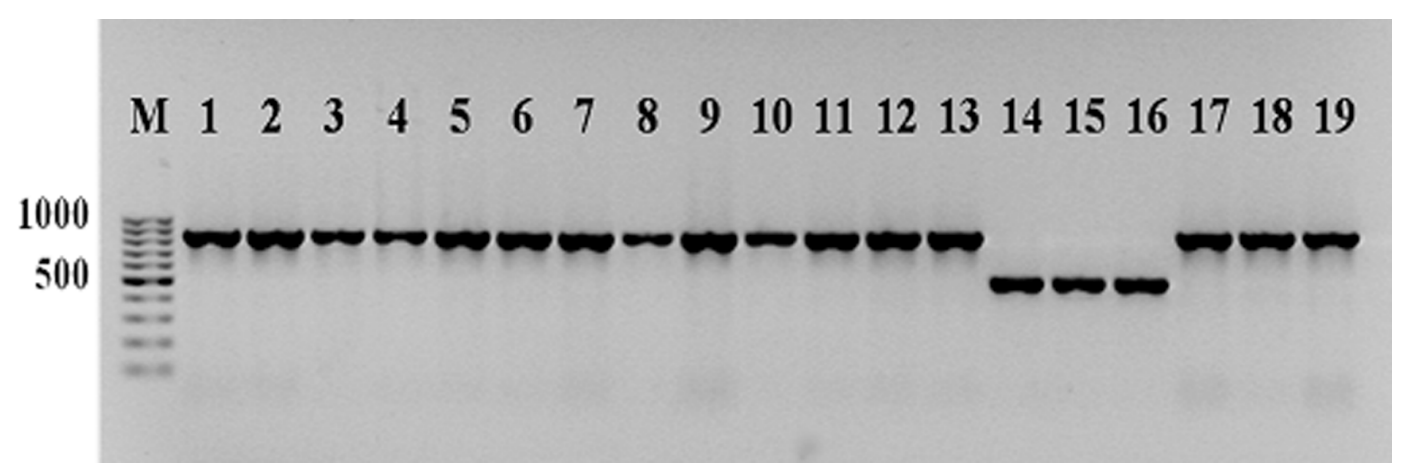

Figure 1. PCR amplification results with tF/ tR SCAR marker. Lines: 1,2 - LA3134, 3,4 - LA2529, 5,6,7 LA2374, 8, 9,10- LA3899, 11,12,13 - LA4062, 14,15,16 - LA3183, 17,18,19 - LA348.
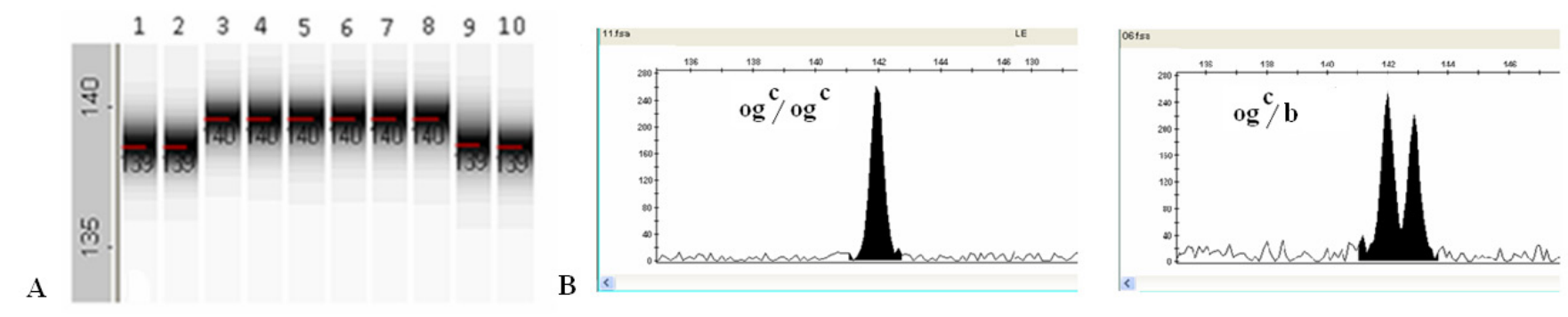

Figure 2. $P C R$ amplification results with $\mathrm{Og}^{\mathrm{c}} \mathrm{F} / \mathrm{Og}^{\mathrm{c}} \mathrm{R}$ SCAR marker. $\mathrm{A}$ - Polyacrylamide gel. Lines: 1-2, 9-10 - with $O g^{c}$ allele, B fragment analysis on an automatic sequencer: homo- and heterozygous samples.
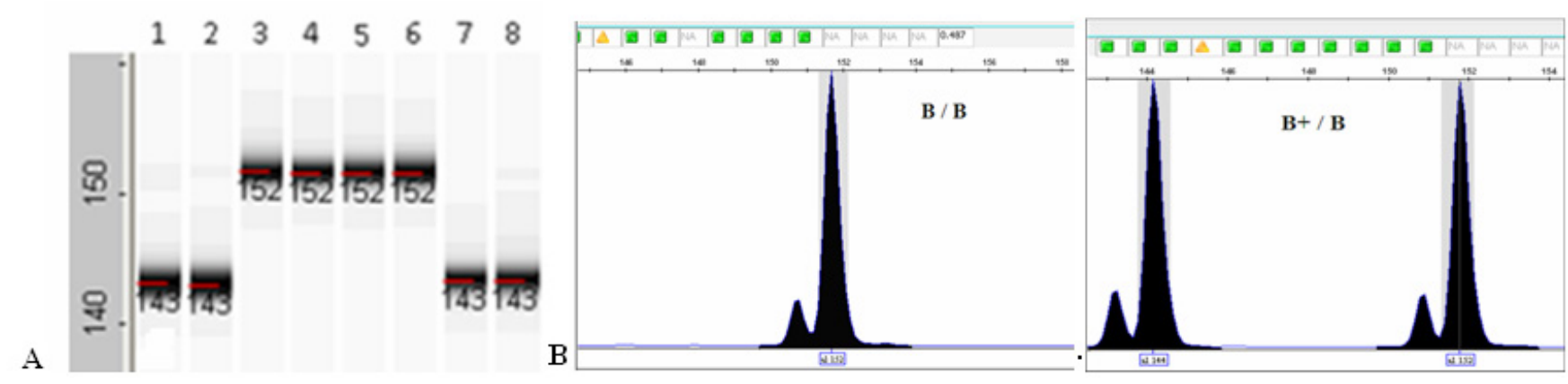

Figure 3. PCR amplification results with BpromF/BpromR SCAR marker. A - Polyacrylamide gel. Lines: 1-2, 7-8 - with $b$ allele, 3-4, 5-6 - with $B$ allele $B$ - fragment analysis on an automatic sequencer: homo- and heterozygous samples.

to an increased prolycopene concentration and generation of fruits with a deep yellow coloring (2).

In order to identify $t$ allele in the gene CRTISO we developed SCAR marker $\mathrm{tF} / \mathrm{tR}$. The primers for this marker were: $\mathrm{tF}$ : ACCAATGCTCCGAATGA, tR: TGGGCTCCCTGGAAAGT (primer annealing temperature $50^{\circ} \mathrm{C}$ ). As you can see from Fig.1, two fragments - $508 \mathrm{bp}$ (wild type allele) and $856 \mathrm{bp}(t$ allele) were obtained as a result of amplification.

Fruits with the old-gold crimson $\left(\mathrm{og}^{\mathrm{c}}\right)$ allele of the CYCB gene are characterized by a high content of lycopene. Such plants have dark red fruits and brown-orange flowers (3). In order to identify $o^{c}$ allele, we developed the SCAR marker $-\mathrm{Og}^{\mathrm{c}} \mathrm{F} / \mathrm{Og}^{\mathrm{c}} \mathrm{R}$. The primers for this marker were: $\mathrm{Og}^{\mathrm{c} F}$ : TAGGTCTATTTTCCAACAA, Og ${ }^{c}$ : AAGACTCTGGCTTTGATG (the primer annealing temperature to $47^{\circ} \mathrm{C}$ ). As a result of PCR amplification, two fragments were obtained - $140 \mathrm{bp}$ (wild type allele) and $139 \mathrm{bp}$ ( og allele) (Fig.2).

The presence of a dominant allele $B$ (Beta carotene) leads to an increased content of $\beta$-carotene and an orange-red color of the fruit pulp (4).

In order to identify $B$ allele, we developed SCAR marker BpromF/BpromR. The primers for this marker were: BpromF: CTATGTTTGTTAGTGCTTGG, BpromR: GAAAATTGTTCATGTGCC (the primer annealing temperature to $55^{\circ} \mathrm{C}$ ).

As a result of PCR amplification, two fragments were obtained in S. lycopersicum genotypes: with a $b$ recessive allele $-143 \mathrm{bp}$, with dominant $B$ allele - 152 bp (Fig.3).

Plants with rin, nor alleles of regulatory genes are characterized by a low level of respiration, weak ethylene synthesis, low activity 


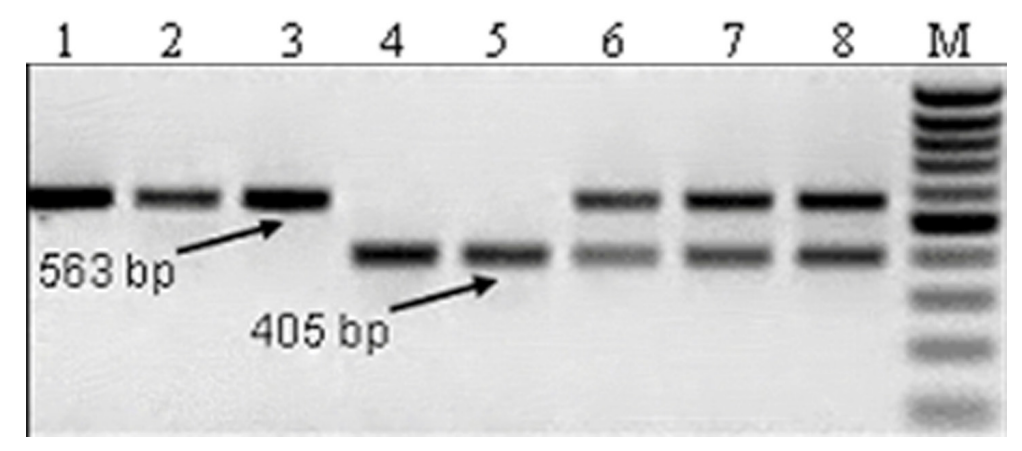

Figure 4. PCR amplification results with the SCAR marker RinF1/ RinwR1/ RinmR2. Lines: 1-3 - L154, 4,5 - Mo 577 (rin), 6-8 -F hybrid (L154 x Mo 577).
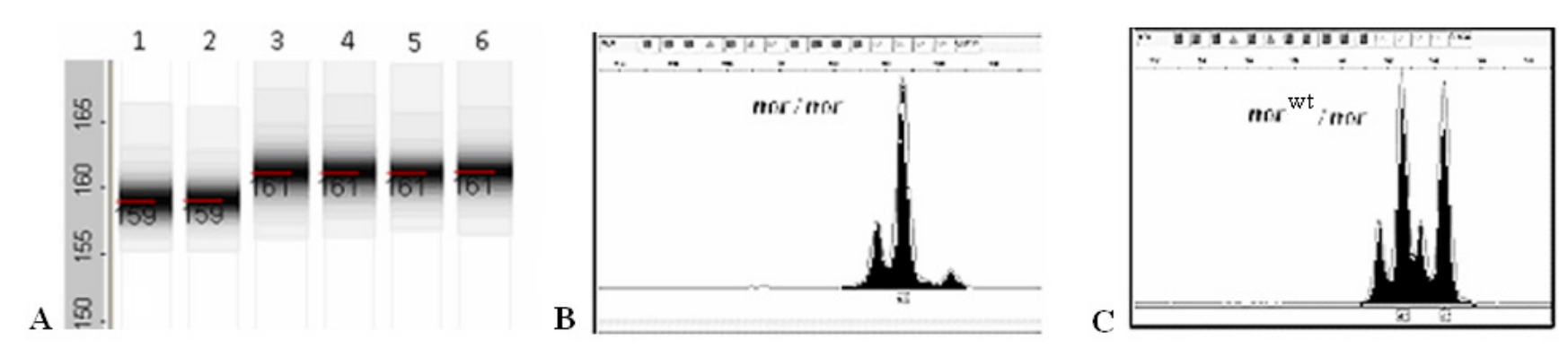

Figure 5. Results of PCR amplification of DNA tomato forms with NorF/ NorR SCAR marker: 1, 2 - Mo 948 (nor), 3, 4 - Mo 950 (nor ${ }^{A}$ ), 5, 6 - Line 19/1 (nor $\left.{ }^{w t}\right)(A)$; Results of fragment analysis of plants F2: homozygous plant (nor/nor) (B) and heterozygous plant (nor/nor $\left.{ }^{w t}\right)(C)$.

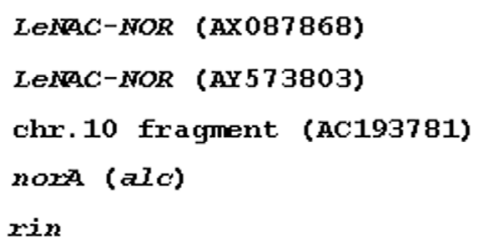

Figure 6. A fragment of the DNA-sequence of the second exon of the LeNAC-NOR gene in the nor ${ }^{A}$ mutant and the wild allele. The arrow shows the position of the point mutation in the nor allele.t (nor/nor $\left.{ }^{w t}\right)(C)$.

of polygalacturonase, a low content of carotenoids and a long period of maturation. They have a blending inheritance in $\mathrm{F}_{1}$ and can be successfully used to develop heterotic tomato hybrids with a prolonged period of fruit storability $(5,6)$.

In order to identify a rin allele, we developed the SCAR marker - RinF1/ RinwR1/ RinmR2. The primers for this marker were: RinF1: TGAACAGCCTTCTCATCATG, RinwR1: ATTCAAAGCATCCATCCAGG, RinmR2: ATTCCATATCAAAGTGAGTAGT (the primer annealing temperature to $55^{\circ} \mathrm{C}$ ).

As you can see from Fig. 4, line Mo577 was a rin mutant form and $\mathrm{F}_{1}$ hybrid L154 x Mo 577 was a rin-heterozygous form.

Inordertoidentifyalleliccomposition of norgene, wedeveloped the SCAR marker - norF/norR. The primers for this norF/norR SCAR marker were NorF: ATGATTGGGTTTTATGTCG;
NorR: CGATTCATTTTCGAGCAA (the primer annealing temperature to $50{ }^{\circ} \mathrm{C}$ ). As a result of PCR amplification (Fig.5), two fragments were obtained: $159 \mathrm{bp}$ (nor allele) and $161 \mathrm{bp}$ (wild type allele).

In the literature, there were some contradictory opinions about the alcobaça mutant inheritance. Mustchler (7) described it as a recessive gene (alc) on chromosome 10, non-allelic to a nor gene, and located at a distance of $17 \mathrm{cM}$ from the latter. As opposed, Lobo et al. (8) described it as another allele in the nor locus, different from nor and nor ${ }^{w t}$ and denoted it by the symbol of $n o r^{A}$. S.V. Malyshev et al. (9), as a result of LeNAC-NOR gene sequencing in the selection line carrying the alc gene, the nor ${ }^{A}$ gene was shown to be a new allelic form of the LeNACNOR gene (Fig. 6). The authors identified a point mutation in the second exon (the replacement of thymine with adenine), leading to an amino acid substitution of valine-asparagine. 


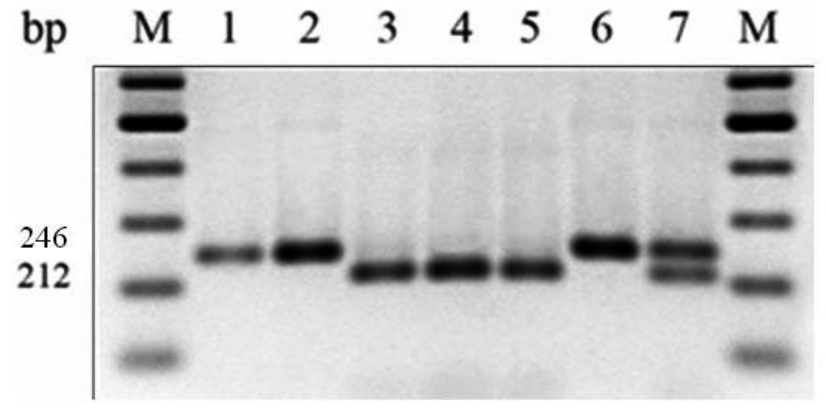

Figure 7. Restriction products of the CAPS marker (dAlc-BspMII) used for nor ${ }^{A}$ allele identification. Lines:1 - La 0059 (nor $\left.{ }^{w t}\right), 2$ Mo 948 (nor), 3 - Mo $950\left(\right.$ nor $\left.^{A}\right), 4$ - La2529 $\left(\right.$ nor $\left.^{A}\right), 5$ - La3134 $\left(\right.$ nor $\left.^{A}\right), 6-$ Mo 577 (rin), $7-\mathrm{F}_{1}$ nor $^{\mathrm{A}} /$ nor $^{\text {wt }}$.

The mutation in the LeNAC-NOR gene of the nor ${ }^{A}$ mutant affects the conserved amino acid in $\mathrm{C}$ subdomain of NAC domain that probably leads to disruption of the $\beta$-layer folding in the domain. This change, respectively, leads to a violation of the protein interaction with the DNA molecule and, probably, a partial loss of protein function and slowing down of the processes associated with maturation. The obtained alc gene sequence was submitted to the GeneBank database under FJ404469 number.

In order to identify a nor ${ }^{A}$ allele, we developed the dCAPS marker - dAlc-BspMII. The primers for the norF/norR SCAR marker were:

F:GTAAAACGACGGCCAGTTACCGGAACCGACAATCCG,
R:TCGATGCAAAATTTAGGAC (the primer annealing temperature to $50^{\circ} \mathrm{C}$ ).

As a result of the amplification with this marker, an amplicon with a length of $246 \mathrm{bp}$ is synthesized. The amplification products are restricted by BspMII endonuclease, the wild allele does not split into fragments, and the nor ${ }^{A}$ mutant allele produces 212 and 34 bp fragments. As you can see from Fig. 7, the lines Mo 950, La2529, and La3134 carry the nor ${ }^{A}$ allele in the homozygous state, and the hybrid $\mathrm{F}_{1}$ nor ${ }^{A} /$ nor $^{w t}$ is heterozygous by the analyzed character.

Five mutant alleles of the $g f$ gene are known from literature sources (10). Green flesh-3 (gf-3) mutation is most common in our tomato collection.

In order to identify a $g f-3$ allele, we developed gf-3F/ gf-3R SCAR marker. The primers for norF/norR SCAR marker were: gf-3F: CCAGGAAAGTTGCCAAGAAC, gf3R: GAAAATTGGAATCGCCAAGA (the primer annealing temperature to $55^{\circ} \mathrm{C}$ ). As a result of PCR amplification, two fragments were obtained: $326 \mathrm{bp}-(g f-3$ allele $)$ and $328 \mathrm{bp}$ (other type of alleles) (Fig. 8).

In order to identify $h p-2^{d g}$ allele, we used CAPS marker TDM2 F1/ TD-M2 R1 developed by Levin et al. (11). The primers for this marker were: TD-M2 F1: TTC TTCGGATTGTCCATGGT, TD-M2 R1: CACCAATGCTATGTGCCAAA (the primer annealing temperature to $55^{\circ} \mathrm{C}$ ). As a result of PCR with the indicated marker, an amplicon measuring $697 \mathrm{bp}$ is synthesized. After the restriction with AclI endonuclease, the mutant allele remains unchanged and other alleles are cut into fragments of $578 \mathrm{bp}$ and $119 \mathrm{bp}$ in size (Fig. 9).
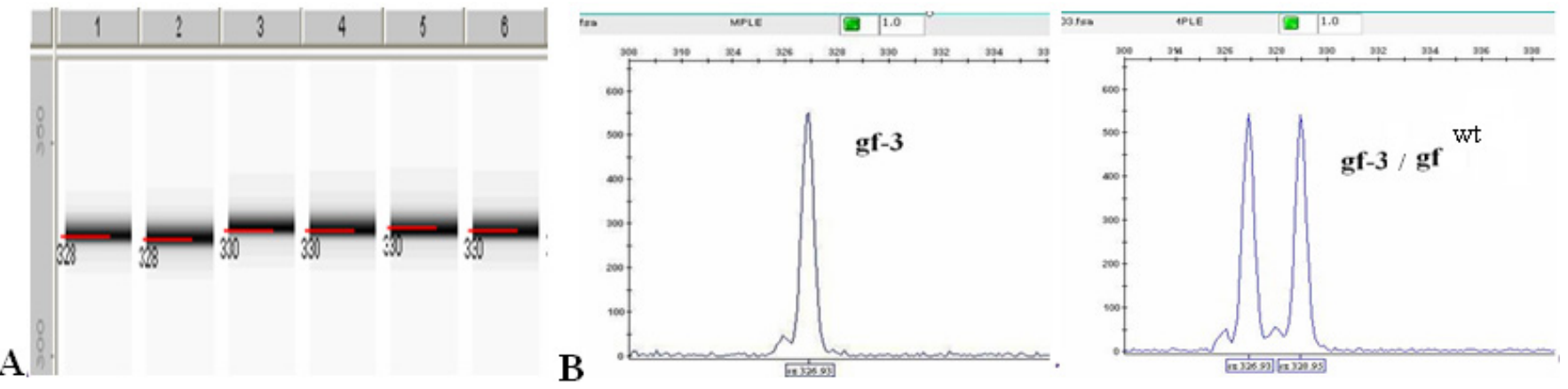

Figure 8. The results of DNA amplification of tomato genotypes with the gf-3F/gf-3R marker: A. Polyacrylamide gel. Lines: 1,2 Black Prince ( $g f-3), 3$, 4 - Black cherry, 5, 6 - Noire charbonneuse; B. Fragment analysis on an automatic sequencer: homo- and heterozygous samples.

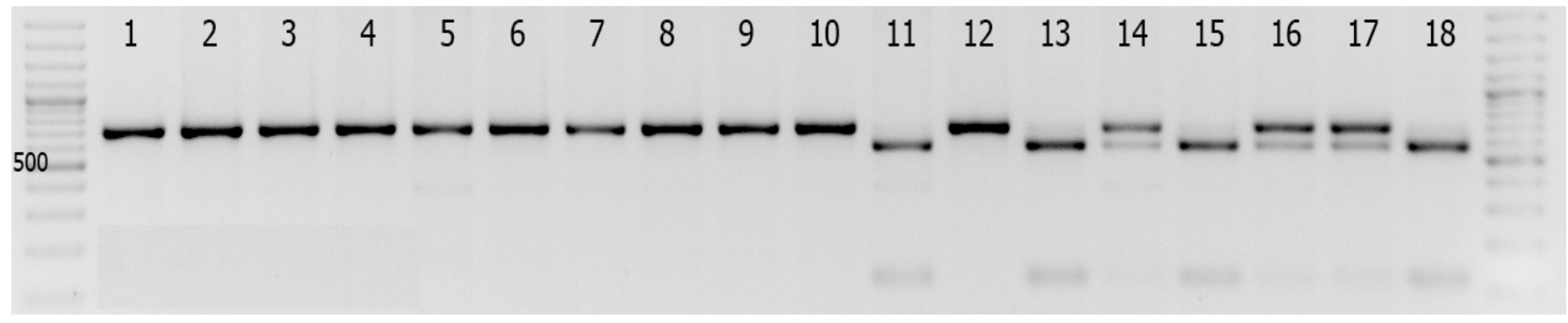

Figure 9. The results of DNA amplification of tomato in $\mathrm{F}_{3}$ population genotypes with $\mathrm{hp}-\mathrm{2}^{\mathrm{dg}} \mathrm{F} / \mathrm{hp}-2^{\mathrm{dg}} \mathrm{R}$ and subsequent restriction by Acll. № 1-10, 12 - homozygous $h p-2^{d g}$ form, No. 14, 16, 17 - heterozygous form, No. 11, 13, 18 - samples with wild allele. 

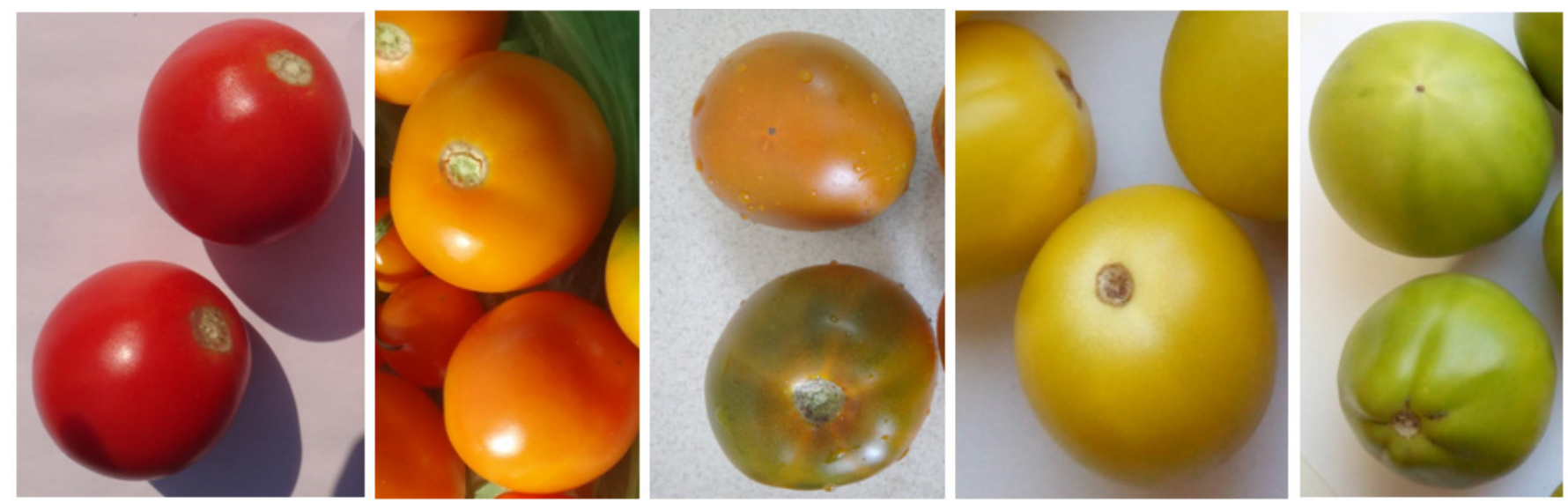

Figure 10. Forms of tomato with various structural and regulatory genes: og $/ / h p 2^{d g}, B / h p 2^{d g}, B / g f-3, B / h p 2^{d g} / n o r, o g c / g f-3 / r i n$.

Table 1. The content of carotenoids in tomato samples with different combinations of quality alleles (mg/100 g wet basis), 2016

\begin{tabular}{|c|c|c|c|c|c|}
\hline \multirow{2}{*}{ Allele combinations } & \multirow{2}{*}{$\begin{array}{c}\beta- \\
\text { carotene }\end{array}$} & \multirow{2}{*}{ Lycopene } & \multirow{2}{*}{ Lutein } & \multicolumn{2}{|c|}{ Chlorophyll } \\
\hline & & & & a & $\mathrm{b}$ \\
\hline $\operatorname{og}^{c} / \operatorname{og}^{c} / / \operatorname{rin}^{w t} / \operatorname{rin}^{w t}$ & 0.85 & 11.0 & 0 & 0 & 0 \\
\hline ogc/ogc//rin/rin & 0.44 & 0 & 0.26 & 0 & 0 \\
\hline $\operatorname{og}^{c} / \operatorname{og}^{c} / / g f-3 / g f-3 / /$ rin $^{w t} /$ rin $^{w t}$ & 0.55 & 5.75 & 0 & 0 & 0 \\
\hline $\operatorname{og}^{c} / \operatorname{og}^{c} / / g f-3 / g f-3 / /$ rin/rin & 0,25 & 0.8 & 0 & 0 & 0 \\
\hline$o g^{c} / o g^{c} / / h p 2^{d q} / h p 2^{d g} / /$ rin $^{w t} /$ rin $^{w t}$ & 1.05 & 16.9 & 2.2 & 0 & 0 \\
\hline $\operatorname{og}{ }^{c} / \operatorname{og} c / / h p 2^{d g} / h p 2^{d g} / /$ rin/rin & 0.7 & 0 & 1.1 & 0.60 & 0.62 \\
\hline $\operatorname{og}^{c} / \operatorname{og}^{c} / /$ nor $^{w t} /$ nor $^{w t}$ & 1.15 & 8.7 & 0 & 0 & 0 \\
\hline ogc/ogc//nor/nor & 0.7 & 1.9 & 0 & 0 & 0 \\
\hline ogc $/$ og $c / / g f-3 / g f-3 / /$ nor/nor & 0.83 & 3.4 & 0.57 & 0.28 & 0.28 \\
\hline$o g^{c} / o g^{c} / / h p 2^{d g} / h p 2^{d g} / /$ nor $^{w t} /$ nor $^{w t}$ & 1.7 & 19.9 & 2.2 & 0 & 0 \\
\hline $\operatorname{og}^{c} / o g^{c} / / h p 2^{d g} / h p 2^{d g} / /$ nor/nor & 1.4 & 0.7 & 1.4 & 0 & 0 \\
\hline$B / B / / r i n^{w t} / r i n^{w t}$ & 2.9 & 1.0 & 1.2 & 0 & 0 \\
\hline$B / B / /$ rin/rin & 0.69 & 0 & 0.6 & 0 & 0 \\
\hline$B / B / / g f-3 / g f-3 / / r i n^{w t} / r^{w} n^{w t}$ & 3.1 & 2.1 & 0 & 0 & 0 \\
\hline$B / B / / g f-3 / g f-3 / /$ rin/rin & 0.6 & 0 & 0.25 & 0.87 & 0.93 \\
\hline$B / B / / h p 2^{d g} / h p 2^{d g} / / r i n^{w t} / \operatorname{rin}^{w t}$ & 5.5 & 0 & 2.15 & 0 & 0 \\
\hline$B / B / / h p 2^{d g} / h p 2^{d g} / /$ rin/rin & 0.6 & 0 & 0.7 & 0.68 & 0.63 \\
\hline$B / B / /$ nor $^{w t} /$ nor $^{w t}$ & 4.7 & 1.43 & 0.75 & 0 & 0 \\
\hline$B / B / / g f-3 / g f-3 / /$ nor $^{w t} /$ nor $^{w t}$ & 1.8 & 5.5 & 1.0 & 3.0 & 2.1 \\
\hline$B / B / / g f-3 / g f-3 / /$ nor/nor & 0.28 & 0 & 0 & 2.3 & 2.6 \\
\hline$B / B / / h p 2^{d g} / h p 2^{d g} / /$ nor $^{w t} /$ nor $^{w t}$ & 5.1 & 0 & 1.0 & 0 & 0 \\
\hline$B / B / / h p 2^{d g} / h p 2^{d g} / /$ nor/nor & 1.63 & 0.3 & 1.2 & 1.01 & 0.80 \\
\hline
\end{tabular}




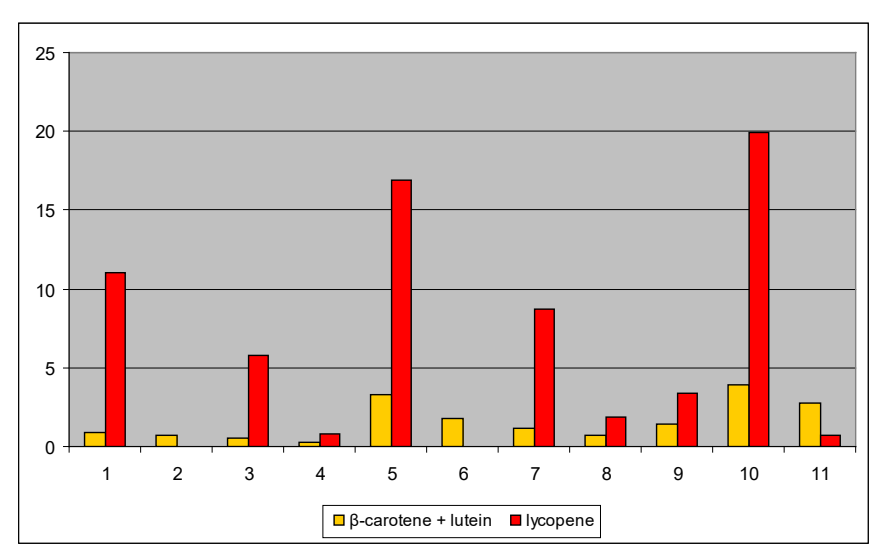

Figure 11. Peculiarities of the carotenoid accumulation in tomato forms with $\mathrm{og}^{c}$ allele. Genotypes of the analysed tomato

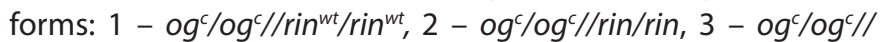

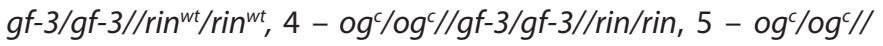

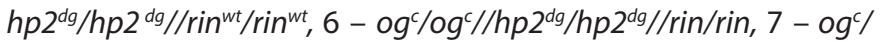
ogc/nor wt/norwt, 8 - ogc/ogc/nor/nor, 9 - ogc/ogc//gf-3/gf-3//nor/

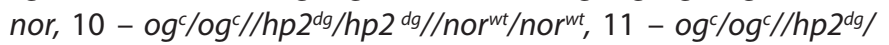
$h p 2^{d g} / /$ nor/nor.

Using the designed methods, the parental forms were selected and $F_{1}$ hybrids with genes involved in the process of biosynthesis and transformation of carotenoids $\left(t, B, o g^{c}\right)$ and genes controlling the ripening of fruits (nor, nor ${ }^{A}$, rin) were developed. After the selection of homozygotes by two pairs of alleles, crossings of the lines with forms containing $g f-3$ and $h p 2^{d g}$ alleles were performed. We earlier presented the development of hybrids and their evaluation (1). Fig. 10-12 and Tables 1-2 show the results of the accumulation of carotenoids in the developed samples with different combinations of alleles in a homozygous form.

Along with these forms, we studied the accumulation of carotenoids in the samples with combinations of genes $t / t / /$ nor ${ }^{w t} /$ nor $^{w t}, t / t / /$ nor $^{A} /$ nor $^{A}, t / t / /$ nor/nor. According to the data obtained, the main fruit carotenoids were neurosporin and $\zeta$-carotene (Table 2). The form with a combination of alleles $t / t / /$ nor $^{A} /$ nor $^{A}$ had, along with a high content of carotenoids, a sufficiently long shelf life, which makes it promising in the accumulation of this type of carotenoids.

Table 2. Carotenoid content in tomato samples with tangerine and non-ripening alleles (mg/100 g wet basis), 2016

\section{Allele}

combinations

\section{neurosporin}

$\zeta$ - carotene

$\begin{array}{lcc}t / t / / \text { nor }^{w t} / \text { nor }^{w t} & 11.6 & 0 \\ t / t / / \text { nor }^{A} / \text { nor }^{\mathrm{A}} & 0 & 7.2 \\ t / t / / \text { nor/nor } & 1.94 & 0.94\end{array}$

\section{Discussion}

The carotenoids' accumulation peculiarities in tomato fruits were studied using different allele combinations of the structural

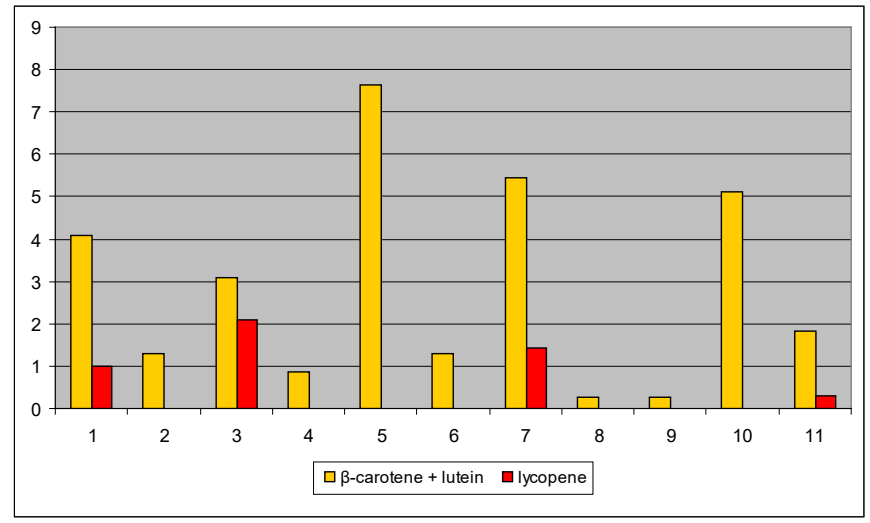

Figure 12. Peculiarities of the carotenoid accumulation in tomato forms with $B$ allele. Genotypes of the analysed tomato forms: 1 - B/B//rin wt/rin wt, $2-B / B / /$ rin/rin, $3-B / B / / g f-3 / g f-3 / /$ rin $^{w t} /$ rin $^{\text {wt }}, 4-B / B / / g f-3 / g f-3 / /$ rin/rin, $5-B / B / / h p 2^{d g} / h p 2^{d g} / /$ rin $^{w t} /$ rin $^{w t}, 6$ - $B / B / / h p 2^{d g} / h p 2^{d g} / /$ rin/rin, $7-B / B / /$ nor $^{w t} /$ nor $^{w t}, 8-B / B / /$ nor/nor, 9-B/B//gf-3/gf-3//nor/nor, $10-B / B / / h p 2^{d g} / h p 2^{d g} / /$ nor $^{\text {wt }} /$ nor $^{\text {wtt }}, 11$ $-B / B / / h p 2^{d g} / h p 2^{d g} / /$ nor/nor.

genes of carotenoid isomerase (CRTISO) and chromoplastspecific lycopene-b-cyclase (CYC-B) and regulatory genes of a long maturation period: (nonripening, ripening inhibitor), the genes increasing the number and size of plastids (high pigment 2 dark green) and modifying the process of chlorophyll destruction (green flesh-3).

As a result of the analysis, we confirmed that fruit shelf life alleles in the homozygous state significantly decrease the accumulation of carotenoids and revealed the following peculiarities of pigment accumulation in tomato fruits: despite the decrease in the accumulation of carotenoids under the effect of rin, nor, nor ${ }^{A}$ genes, their combination in the genotype in the heterozygous state with $B, o g^{c}, t, h p$ genes in the homo- and heterozygous state restores the accumulation of carotenoids in tomato fruits to the level of standards - the forms with no storability genes.

The $h p 2^{d g}$ allele increases the accumulation of carotenoids determined by the main structural genes, a combination of $\mathrm{og}^{c} /$ $h p 2^{d g}$ alleles provides the maximal lycopene accumulation and $B / h p 2^{d g}$ - $\beta$-carotene accumulation in tomato fruits.

Using efficient molecular genetic methods and traditional tomato breeding techniques, we have developed the homozygous forms that constitute valuable material in fruit quality tomato breeding in Belarus.

\section{Conclusions}

In the fruit quality breeding process, we recommend to use the samples with carotenoid content genes $\left(\operatorname{og}^{c} / h p 2^{d g}, B / h p 2^{d g}\right)$ as maternal forms and as paternal forms - the samples with comlex fruit quality genes $\left(o g^{c} / h p 2^{d g} / n o r, o g^{c} / h p 2^{d g} /\right.$ rin, $B / h p 2^{d g}$ /nor, B/hp2 $2^{d g} / \mathrm{rin}$, ogc/gf-3/nor, ogc/gf-3/rin, B/gf-3/rin, B/gf-3/ nor). Appliing of such forms will allow to increase the content of certain carotenoids by a factor of 2-2.5, which in turn enhances useful properties (antioxidant and other) of fruits and creates their value as healthy food. 


\section{Acknowledgments}

We thank the C.M. Rick Tomato Genetics Resource Center (University of California, Davis) for the seed accessions; the All-Russian Research Institute of Vegetable Breeding and Seed Production (ARRIVBSP) for the biochemical analysis of fruits.

\section{Conflict of interest statement}

The authors declare that they have no conflict of interest.

\section{References}

1. Adzhieva VF, Babak OG, Shoeva OY, Kilchevsky AV, Khlestkina EK. Molecular genetic mechanisms of the development of fruit and seed coloration in plants. Russian Journal of Genetics: Applied Research. 2016; 6(5):537-52.

2. Eckardt NA. Tangerine dreams: cloning of carotenoid isomerase from Arabidopsis and tomato. Plant Cell. 2002; 14:289-92.

3. Labate JA, Grandillo S, Fulton T., Munos S. et all. Tomato / Genome mapping and molecular breeding in plants. Vol. 5 / Vegetables. Berlin, Heidelberg, New York: Springer. 2007: 1-126.

4. Ronen G, Carmel-Goren L, Zamir D, HirschbergJ. An alternative pathway to b-carotene formation in plant chromoplasts discovered by map-based cloning of Beta and old-gold color mutations in tomato. PNAS. 2000; 97(20):11102-7.

5. Vrebalov J, Ruezinsky D, Padmanabhan V, White R, Medrano D,
Drake R, Schuch W, Giovannoni J. A MADS-box gene necessary for fruit ripening at the tomato ripening-inhibitor (Rin) locus. Science. 2002; 296: 343-5.

6. Gavrish SF, Korol' VG. Some biological features of the tomato hybrids F1 bearing the gene nor. J. Of Izvestia of Timiryazev Agricultural Academy. 1991; 1: 118-32. (In Russian)

7. Mutschler MA. Inheritance and linkage of the 'Alcobaca' ripening mutant in tomato. J. Amer. Soc. Hort. Sci. 1984; 109(4): 500-3.

8. Lobo M; Basset MJ; Hannah LC. Inheritance and characterization of the fruit ripening mutation in „alcobaça" tomato. J. of American Society for Horticultural Science. 1984; 109: 741-5.

9. Kilchevsky AV, Babak, OG, Adzhieva VF, Nekrashevich NA, Malyshev SV, Grushetskaya ZF, Mishin LA, Dobrod'kin MM, Zaitseva IE, Pugacheva IG, Molecular technologies in tomato breeding (Solanum lycopersicum L.) in Geneticheskie osnovy selektsii rastenii (Genetic Basis of Plant Breeding). Minsk, 2014. vol. 4. (In Russian)

10. Barry CS, McQuinn RP, Chung M-Y, Besuden A, Giovannoni JJ. Amino acid substitutions in homologs of the Stay-Green protein are responsible for the green-flesh and chlorophyll retainer mutations of tomato and pepper. Plant Physiol. 2008; 147: 179-87.

11. Levin P, Frankel N, Gilboa S, Tanny A. Lalazar T. The tomato dark green mutation is a novel allele of the tomato homolog of the DEETIOLATED1 gene. Theor Appl Genet. 2003; 106:454-60. 